\title{
CARACTERÍSTICA AERODINÂMICA DA TURBULÊNCIA SOBRE E DENTRO DO DOSSEL DE UMA FLORESTA DE TERRA FIRME NA AMAZÔNIA CENTRAL
}

\author{
Newton Silva de Lima ${ }^{1, *}$, Julio Tóta², Maurício José Alves Bolzan³, \\ Roseilson do Vale ${ }^{1}$, Raoni Santana ${ }^{1}$ \\ ${ }^{1}$ Doutorando em Clima e Ambiente - INPA/UEA/ULBRA \\ ${ }^{2}$ Universidade Federal do Oeste do Pará - UFOPA/CLIAMB \\ ${ }^{3}$ Universidade Federal de Góias - Jataí - UFG \\ *newtonulbra@gmail.com
}

\section{RESUMO}

Observações micrometeorológicas realizadas por anemômetros ultrassônicos do escoamento atmosférico na estrutura física de uma floresta de terra firme na Amazônia central no experimento ATTO-CLAIRE (POI-1/2012), foram utilizadas para caracterizar a turbulência sobre e dentro do dossel, com medidas em dez níveis a partir da superfície ( $23 \mathrm{~m} ; 3 \mathrm{~m} ; 36 \mathrm{~m} ; 41 \mathrm{~m} ; 45 \mathrm{~m} ; 50 \mathrm{~m} ; 57 \mathrm{~m} ; 62 \mathrm{~m} ; 70 \mathrm{~m} ; 78 \mathrm{~m}$ ). O método proposto foi de estatística de Análise de Quadrante, Espectro Global de Ondeleta para a determinação natureza da turbulência. Foram observadas Estruturas Coerentes e Turbulência de 3 e 2 dimensões para condições instável e estável da atmosfera. A turbulência foi caracterizada de quase-duas dimensões e associada à Enstrofia.

Palavras-chave: ATTO-CLAIRE, análise de quadrante, espectro global de ondeleta

\begin{abstract}
Micrometeorological observations made by ultrasonic anemometers of the atmospheric flow in the physical structure of an upland forest in central Amazonia in the experiment ATTO-CLAIRE (IOP-1/2012) were used to characterize the turbulence on or within the canopy, with measurements in ten levels from the surface $(23 \mathrm{~m}, 30 \mathrm{~m}, 36 \mathrm{~m}, 41 \mathrm{~m}, 45$ m, $50 \mathrm{~m}, 57 \mathrm{~m}, 62 \mathrm{~m}, 70 \mathrm{~m}, 78 \mathrm{~m}$.). The proposed method was the statistical Quadrant Analysis, Global Wavelet Spectrum in order to determine the nature of turbulence. We observed Coherent Structures and turbulence 3D and 2D for stable and unstable atmosphere of the conditions. We characterized in quasi-2D turbulence and Enstrophy associated.
\end{abstract}


Keywords: ATTO-CLAIRE, quadrant analysis, global wavelet spectrum.

\section{INTRODUÇÃO}

$\mathrm{Na}$ caracterização dos processos de transporte turbulento de momentum, calor e massa na camada de ar imediatamente acima e dentro de vegetação densa e alta é fundamental conhecer a estrutura física e aerodinâmica da superfície da floresta e subbosque, que são regiões onde complexos processos micrometeorológicos ocorrem e que foram observados por Foken (2008; 2012). Porém, parametrizar estas características constitui-se em tarefa árdua e de diferentes formulações empíricas para determinadas estruturas de terrenos (Raupach et al. 1996; Lyra e Pereira, 2007).

\section{MATERIAL E MÉTODOS}

\section{O SÍTIO EXPERIMENTAL}

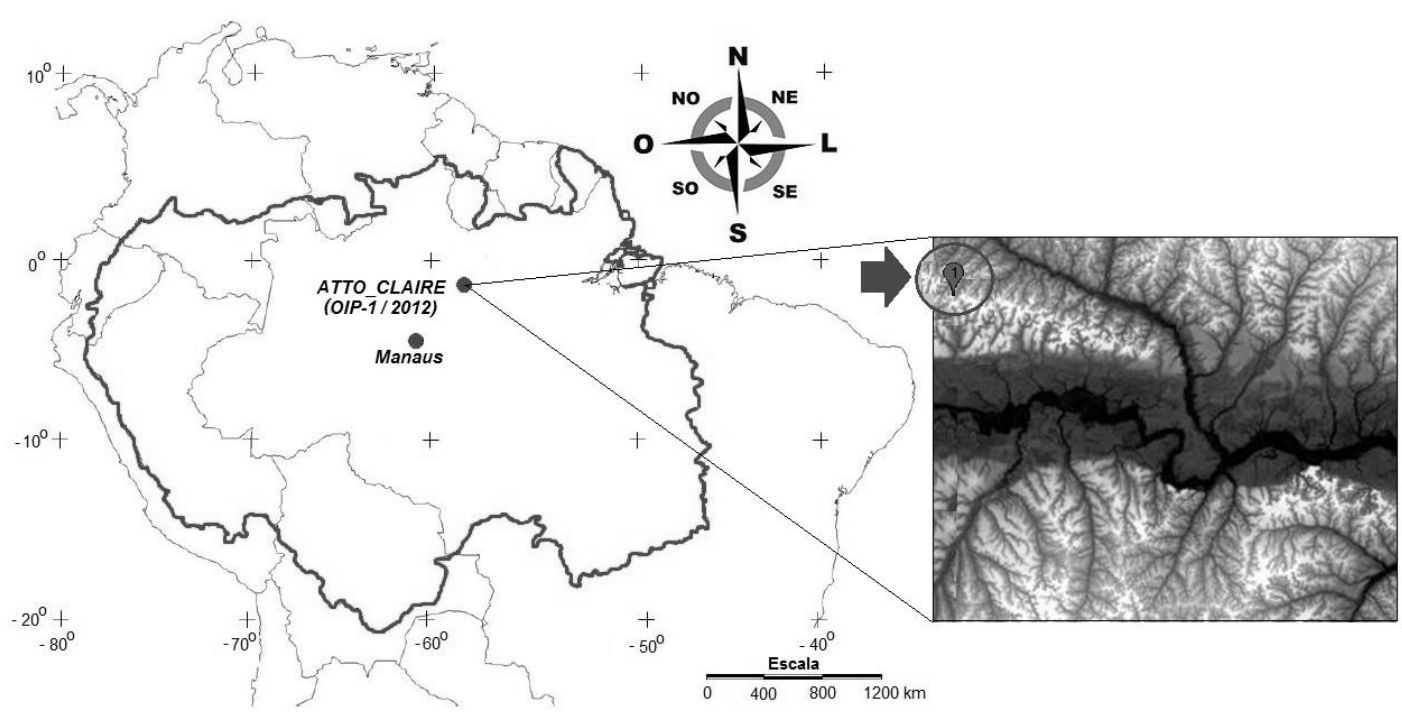

Fig. 1. Platô do sítio experimental ATTO-CLAIRE (IOP-1 / 2012) na Reserva de Desenvolvimento Sustentável do Uatumã - AM, dentro da bacia amazônica (circundada). Observa-se na Figura em destaque que a cor mais escura preta (menores elevações) que são os rios, sendo o mais largo o Rio

Uatumã e a cor mais clara (maiores elevações). (Fonte: UEA - INPA - LBA - MAX PLANCK;

SRTM - USGS-NASA, 2012).

O experimento ATTO-CLAIRE (POI-1/2012) foi desenvolvido durante a estação úmida e interface para estação seca na Amazônia central em um platô, 
(28'32.42”S; 590’3.50”W, ALT $131 \mathrm{~m}$ ), de topografia complexa (Figura 1). O experimento contou com três anemômetros ultrassônicos 3D. A torre com as seguintes características; altura de 80 metros de altura e área de secção transversal triangular de $0,156 \mathrm{~m}^{2}$. Os equipamentos foram posicionados nas seguintes alturas: $78 \mathrm{~m} ; 41 \mathrm{~m}$ e 30 m para o Wind Master; 57 m; 70 m e 62 m para o Met Pack; 23 m; 36 m; 45 m e 50 m para o Wind Sonic. O método das covariâncias foi utilizado para interpretação dos dados.

\section{RESULTADOS E DISCUSSÃO}

O subdomínio de flutuação do número de onda é definido pelo inverso da escala de Ozmidov (faixa de 10 a 100 m) (Stull, 1989) que separa o subdomínio de flutuação (número de onda médio) do subdomínio inercial (número de onda alto).

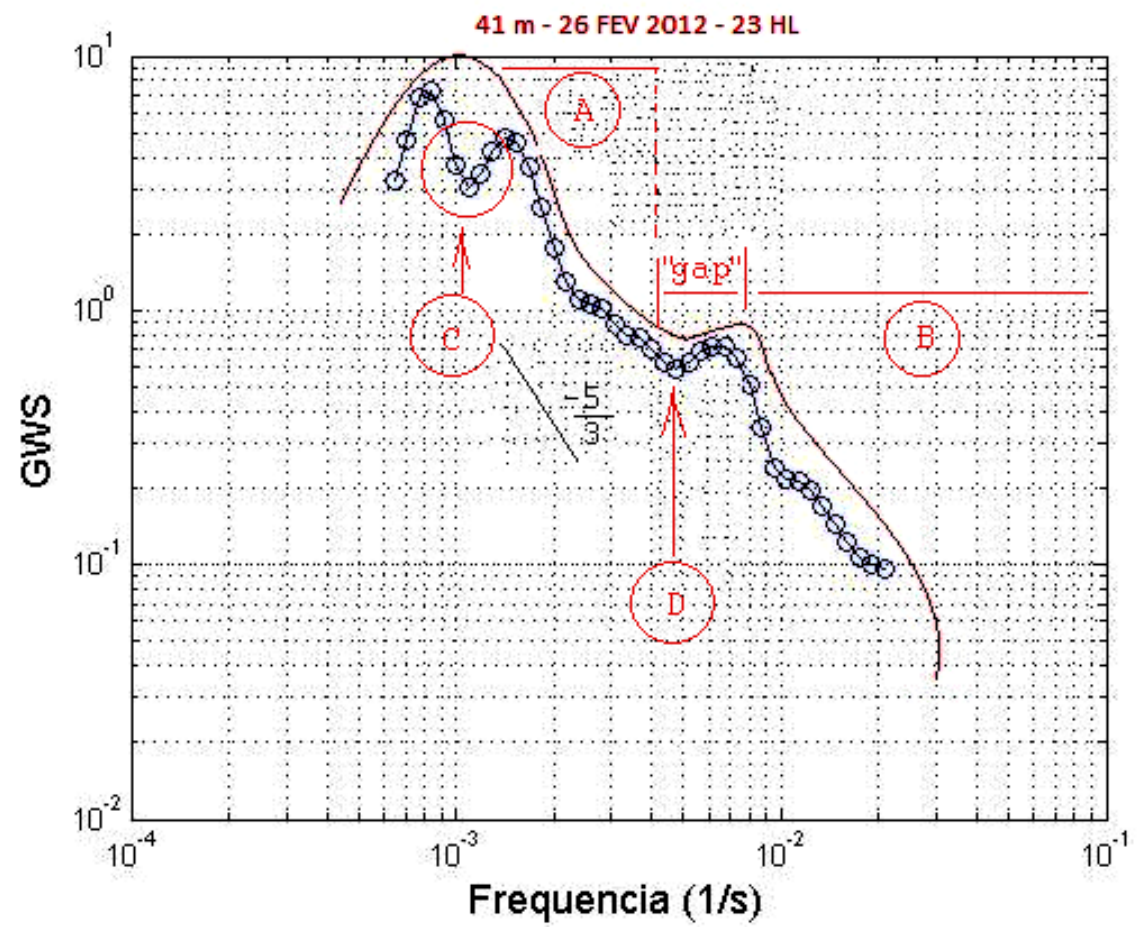

Fig. 2 - Espectro Global de Ondeleta para as variâncias das componentes ( $\left.w^{\prime}\right)$ do vento e da Temperatura $\left(T^{\prime}\right)$ sobre a copa do dossel. $(A)=$ Subdomínio de Flutuação. $(B)=$ Subdomínio Inercial. $(C)$ = Turbulência sem forçante externa, decai em função da dependência do expoente "infravermelho" da turbulência. (D) = Flutuabilidade do Número de Onda. Traço Vermelho ajuste do espectro. Traço Azul espectro real. (Fonte: ATTO-CLAIRE/POI-1, 2012). 
Entre os subdomínios de flutuação e inercial existe um espaço (em inglês "gap", Fig. 2). Este espaço é mais aparente no espectro de temperatura potencial (Stull, 1989). Uma possível explicação segundo Kaimal e Finnigan (1994), é que o "gap" acontece quando a energia transferida do subdomínio de flutuação para o subdomínio inercial é em baixo número de onda e é bloqueada na região $\kappa^{-3}$ sendo assim, conduzida para o pico mais próximo da flutuação do número de onda.

\section{CONCLUSÃO}

A turbulência desenvolvida está mais associada à cascata de Enstrofia e é quase-2D, com predominância de vorticidade. Existe um colapso de onda no espectro de energia, o que indica assinatura de Estrutura Coerente, principalmente sobre a copa. Dentro da copa a turbulência permanece em quase-2D, ou seja no plano. O vento foi predominantemente meridional no intervalo de $\left(\kappa^{-4} \sim \kappa^{-2}\right)$ e zonal entre $\left(\kappa^{-5 / 3} \sim \kappa^{-1}\right)$.

\section{AGRADECIMENTOS}

Gostaríamos de demonstrar nosso reconhecimento ao Programa de Pós-Graduação CLIAMB (LBA-INPA-UEA), FAPEAM, ULBRA Manaus, a UFG, a UFAM e ao Instituto Max Planck. Somos igualmente gratos aos pesquisadores Eliane Alves e Veber Moura (LBA-INPA).

\section{REFERÊNCIAS}

FOKEN, T. 2008. Micrometeorology . Springer-Verlag Berlin Heidelberg, p. 320.

KAIMAL, J.C., FINNIGAN, J.J., “Atmospheric Boundary Layer Flows (Their structure and measurement)” NY, Oxford, OXFORD UNIVERSITY PRESS,1994. p.304.

LYRA, G. B., PEREIRA, A. R. Parâmetros de rugosidade aerodinâmica sobre vegetação esparsa em região semi-árida. Revista Brasileira de Meteorologia, v.22, n.2, 262-272, 2007

RAUPACH, M. R.; FINNIGAN, J.J.; BRUNET, Y., 1996. Coherent eddies and turbulence in vegetation canopies: The mixing-layer analogy, Boundary-Layer Meteorology, v. 78, n. 3-4, p. 351-382.

STULL, R. B. 1988. An Introduction to Boundary Layer Meteorology. Kluwer Academic, 670 p.

WYNGAARD, JOHN C., 2008: Cambridge University Press, Cambridge CB2 8RU, $\mathrm{UK}, 407 \mathrm{p}$. 